\title{
EFFECT OF SOME ANTIOXIDANT FOOD ADDITIVES ON THE DEGRADATION OF CORNELIAN CHERRY ANTHOCYANINS
}

\author{
BIANCA MOLDOVAN ${ }^{\mathrm{a}}$, LUMINIŢA DAVID ${ }^{\mathrm{a}^{*}}$
}

\begin{abstract}
Anthocyanin rich fruits present a great potential as source of natural food colorants. Their use in the food industry is limited by their low stability. The influence of storage media and addition of three widely used antioxidant food additives, such as citric acid, ascorbic acid and butylated hydroxyanisole, on the stability of Cornelian cherry anthocyanins, was investigated at room temperature. In aqueous solutions, the degradation of these valuable pigments occurred slower compared to alcoholic solution. Addition of ascorbic acid significantly enhanced anthocyanins degradation in ethanolic or aqueous solutions, while citric acid and BHA had a positive effect on the anthocyanins' stability in ethanol. The Cornelian cherry anthocyanins' degradation followed first-order reaction kinetics. The obtained kinetic parameters (reaction rate constants $k$ and halflifes $t_{1 / 2}$ ) clearly indicated that Cornelian cherry anthocyanins stored in ethanol in presence of citric acid present the highest stability, the degradation rate constant being $0.74 \cdot 10^{-3} \mathrm{~h}^{-1}$.
\end{abstract}

Keywords: Cornus mas L., antioxidant food additives, anthocyanins, degradation kinetics

\section{INTRODUCTION}

Consumers food choices are strongly influenced by sensory parameters of foods among which colour plays an important role. The slightest changes of this characteristic, which can occur during processing or storage, can affect the people's perception on the quality of food products, the loss of the colour being an important factor in assessing the food quality. The compounds used in the food industry in order to confer vivid colours to products are synthetic dyes which generally imply negative effects on the human health due to their high toxicity. As a consequence, there is a high concern in using synthetic dyes as food colorants and an increased tendency to replace these compounds

a Babeş-Bolyai University, Faculty of Chemistry and Chemical Engineering, 11 Arany Janos str., RO-400028, Cluj-Napoca, Romania

*Corresponding author: muntean@chem.ubbcluj.ro 
with natural pigments. One of the most valuable class of water soluble natural coloured compounds are anthocyanins which can be successfully replace synthetic dyes as a safe alternative. Adding these natural phytocomponents to foods will not only impart vivid colours ranging from red to purple and blue but will also confer functional characteristics to foods due to their wide range of healthy beneficial effects. They can be used as bioactive ingredients in a large number of functional foods as a consequence of their high antioxidant capacity which confer them the potential of reducing the risk of chronic diseases such as cancer, neurodegenerative and cardiovascular diseases. They are also known to possess antimicrobial, anti-diabetic and anti-inflammatory properties [1, 2]. Anthocyanin can be used as versatile tools for material engineering and surface functionalization [3-5]. However, their utilisation as natural food pigments is restricted due to their vulnerability to degradation during processing or storage of food products which mostly leads to an undesirable loss of colour. The anthocyanins' degradation is a complex process which is strongly influenced by many factors such as chemical structure of the pigment, temperature, $\mathrm{pH}$ value, light, oxygen, enzymes and presence of metals [6]. Hence, there is a growing need in finding new sources, plants rich in anthocyanic pigments and investigating their stability in various processing and storage conditions in order to use these compounds as healthy natural colorants in the food industry.

Cornelian cherries are the edible fruits of Cornus mas L. plant original from Middle East and South-Eastern Europe. They are less consumed fresh due to their astringency but are traditionally used as ingredients of jams, marmalades, compotes, vinegars and liquors [7]. The Cornelian cherry fruits are recognised as a rich source of health promoting compounds such as vitamin $\mathrm{C}$ and polyphenols among which anthocyanins are present in significant amount [8-10]. All these compounds are linked with a high range of biological activities reported for Cornus mas fruits such as antioxidant, anti-inflammatory, anticancer and have been traditionally used in the treatment of diarrhea, fever, urinary tract infections, malaria and kidney stones [11-14]. Due to all these biological properties, the chemical composition of Cornelian cherry fruits was intensively investigated. Their anthocyanic profile was established, and various results were reported, depending on cultivars and environmental factors. The 3-glycosylated cyanidin, pelargonidin and delphinidin derivatives were found to be the most common red pigments of Cornelian cherries. Milenckovic Andelkovic and co-workers identified three major anthocyanins: cyanidin-3-galactoside, pelargonidin-3-glucoside and delphinidin-3-galactoside [15] while Moldovan et al. reported the identification and quantification of cyanidin-3-galactoside, pelargonidin-3-glucoside and delphinidin-3-galactoside [16], results in agreement with those of Pawlowska et al. [17]. Using HPLC/DAD and UPLC-ESI/MS techniques, Sozanski et al. identified seven anthocyanins in Cornelian cherry fruits, cyanidin-3-galactoside being the major one [18]. 
Food products regularly contain antioxidant additives responsible for their protection against oxidation, used to increase their shelf life. Among these, the most frequently used antioxidants in food industry are ascorbic acid (AA), phenolic compounds such as tocopherols, butylated hydroxytoluene (BHT) and butylated hydroxyanisole (BHA), which act as free radical scavengers or singlet oxygen quenchers, and citric acid (CA) or EDTA which exert their antioxidant effect by chelating pro oxidative metals [19]. Apart its antioxidant activity, ascorbic acid is also added to food products, especially fruit juices, to increase their nutritional value.

The aim of the present study was to evaluate the influence of some antioxidant food additives, such as AA, BHA and CA on the stability of Cornelian cherries' anthocyanins by storage at room temperature in aqueous and alcoholic media and to determine the kinetic parameters of the degradation process of these compounds in various conditions, in order to use Cornelian cherry fruits anthocyanins as healthy food colorants in fruit derived products and alcoholic beverages.

\section{RESULTS AND DISCUSSION}

Cornelian cherries are red fruits, colour due to the presence of anthocyanins, therefore they can be exploited as a valuable source of anthocyanin pigments. Anthocyanins are known to be degraded or decolorized during food processing and storage. Their stability are influenced by some factors such as chemical structure of the pigment, $\mathrm{pH}$ value, temperature, solvents, oxygen, and presence of ascorbic acid, metal ions and sugars. The presence of a glycosyl moiety and of acyl groups linked to the sugars attached to the pigment molecule positively affects the stability and colour of anthocyanins. The position of these substituents on the anthocyanin skeleton was proved to be important for the stabilisation effect. The loss of attached sugars results in a rapid decomposition and irreversible loss of colour, due to a higher reactivity of non-glycosylated compounds. Although at room temperature anthocyanins are quite stable at acidic $\mathrm{pH}$, they rapidly degrade at elevated temperatures or alkaline media. The reaction of orto-diphenolic groups with metal ions (iron, copper, aluminium, tin) leads to the formation of stable red, blue and violet metal chelates, but the use of these metal complexes in the food industry is still limited [20]. Ascorbic acid was found to significantly enhance anthocyanins' degradation, especially at elevated temperatures, possible as a result of condensation reaction between ascorbic acid and anthocyanins. A free radical mechanism for this deterioration process was also proposed, involving an oxidative cleavage reaction of the pyrylium ring. However, the chemical structures of the degradation products of anthocyanins in the presence of ascorbic acid have not yet been determined $[20,21]$. 
In order to isolate these pigments from the fruits, these were subjected to a solid-liquid conventional extraction, using ethanol as solvent. In order to investigate the combined effect of storage solvent and presence of the antioxidant food additives on the stability of anthocyanins isolated from Cornus mas fruits, the variation of the total anthocyanin content (TAC) of the extracts was monitored daily over a period of 8 days of storage in water and ethanol, in the presence of commonly used additives such as $\mathrm{AA}, \mathrm{CA}$ and $\mathrm{BHA}$, at room temperature. The quantitative evaluation of anthocyanins was accomplished by using a widely applied method developed by Giusti and Wrolstad [22], known as the $\mathrm{pH}$ differential method. This method exploits the structural, and hence the colour, dependence of anthocyanins on $\mathrm{pH}$ value. At $\mathrm{pH}=1$, the anthocyanins are present as a red coloured flavylium cation while raising the $\mathrm{pH}$ to 4.5 will lead to structure change into a colourless carbinol. By using UV-Vis spectroscopy, measuring the absorbance of an anthocyanin solution at these two $\mathrm{pH}$ values, allows the determination of anthocyanins' concentration. The total monomeric anthocyanin content (TAC) of the Cornelian cherry fruits extract was assessed by applying this method using equations $(1,2)$ :

$$
T A C=\frac{A \cdot M W \cdot D F \cdot 1000}{\varepsilon \cdot l}
$$

where: $\mathrm{A}=$ absorbance, calculated as: (equation 2 )

$$
A=\left(A_{p H} 1.0-A_{p H} 4.5\right) 517 \mathrm{~nm}-\left(A_{p H} 1.0-A_{p H} 4.5\right) 700 \mathrm{~nm}
$$

$\mathrm{MW}=449.2 \mathrm{~g} / \mathrm{mol}$ (molecular weight of Cy-3-Glu)

$\mathrm{DF}=$ dilution factor

$\mathrm{I}=$ path length $(1 \mathrm{~cm})$

$\varepsilon=26900 \mathrm{~L} / \mathrm{mol} / \mathrm{cm}$ (molar extinction coefficient of Cy-3-Glu)

$1000=$ conversion factor from gram to milligram

The average concentration of monomeric anthocyanins in the Cornelian cherry extract was $23.54 \pm 1.12 \mathrm{mg} / \mathrm{L}$.

The changes of total monomeric anthocyanin concentration were monitored during storage of Cornelian cherry anthocyanins in water and ethanol in the presence of AA, CA and BHA. After 192 hours of storage, the anthocyanin concentration decreased to $77.78 \%$ for the untreated sample stored in ethanol while adding ascorbic acid resulted in a concentration decrease to $73.96 \%$. A more dramatic concentration decrease was observed for the anthocyanins stored in water in the presence of AA, only $41.75 \%$ of the initial TAC being determined after the same time interval. The deleterious effect of 
AA on the anthocyanin stability was already mentioned in other studies [2326]. The presence of AA accelerates anthocyanins' degradation leading to a significant loss of colour during storage due to either the direct condensation of anthocyanins with ascorbic acid or to formation of hydrogen peroxide which cleaves the pyrylium ring by a free radical mechanism $[25,27]$. Our results are in agreement with those obtained in studies investigating anthocyanins' degradation from various sources such as blackcurrant, elderberry, purple sweet potato, pomegranate $[23,26,28]$.

The addition of other food antioxidants such as CA or BHA did not present a similar destabilizing effect on the Cornelian cherry anthocyanins as AA. $\mathrm{CA}$ addition resulted in a slight increase of the anthocyanin stability, compared to sample stored without any antioxidant additive, by storage in ethanol while storage in water resulted in no significant influence of CA on anthocyanin stability. Butylated hydroxyanisole (BHA) enhanced the anthocyanin stability when these pigments were stored in alcohol, while a slight negative effect of this antioxidant additive was observed at water storage, in line with findings of Cata et al. [29] by storage of mulberry anthocyanins.

Anthocyanin degradation is known to follow a first order reaction kinetics [30-33]. Determining the kinetic parameters of this process such as degradation rate constant $(k)$ and half-life value $\left(t_{1 / 2}\right)$ is very important to compare the combined influence of the added food additives and storage solvent on the stability of the anthocyanic pigments. In order to determine these parameters the variation of concentration during storage was fitted to a first order kinetic model. The kinetic plots of anthocyanins storage degradation process in the investigated conditions are given in Figure 1 (storage in water) and Figure 2 (storage in ethanol).

The high values of the determination coefficients $\left(R^{2}>0.95\right.$, Table 1$)$ for all treatments confirm the fact that the first order kinetic model is the most suitable for predicting the Cornelian cherries anthocyanin degradation process. The kinetic rate constants $\mathrm{k}$ for each degradation reaction was calculated using equation (3):

$$
C=C_{0} \exp (-k t)
$$

where: $\mathrm{C}$ and $\mathrm{C}_{0}$ are the total anthocyanin content $(\mathrm{mg} / \mathrm{L})$ at time $\mathrm{t}$ and $\mathrm{t}=0$, respectively

$\mathrm{t}=$ time $(\mathrm{h})$

$\mathrm{k}=$ rate constant $\left(\mathrm{h}^{-1}\right)$ 


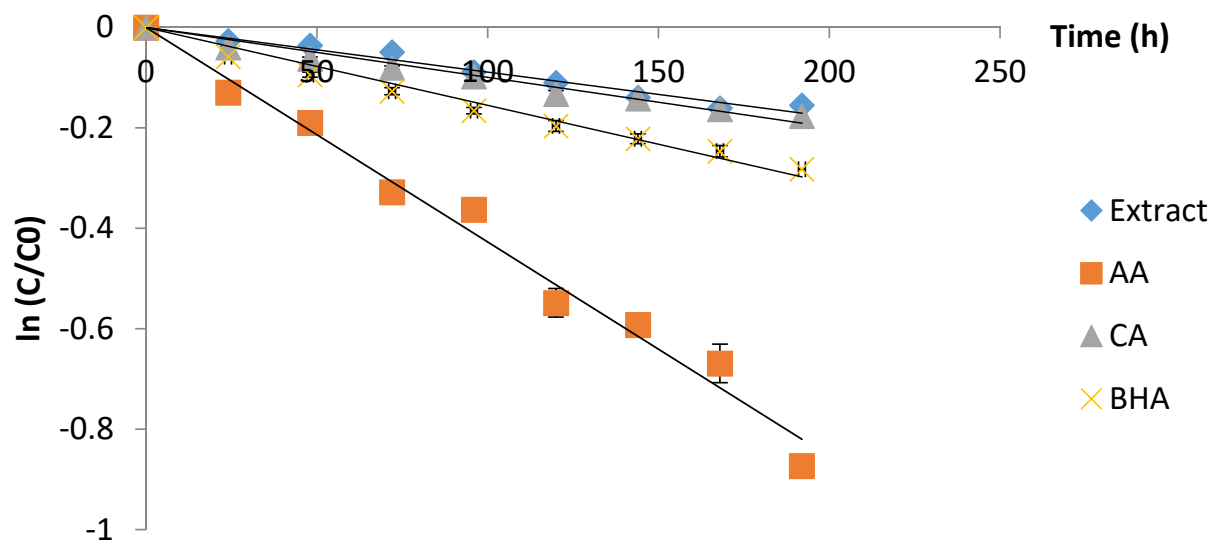

Figure 1. The degradation process of Cornelian cherries anthocyanins stored in water. Error bars represent standard deviation $(n=3)$.

In the some cases they are smaller than the symbol.

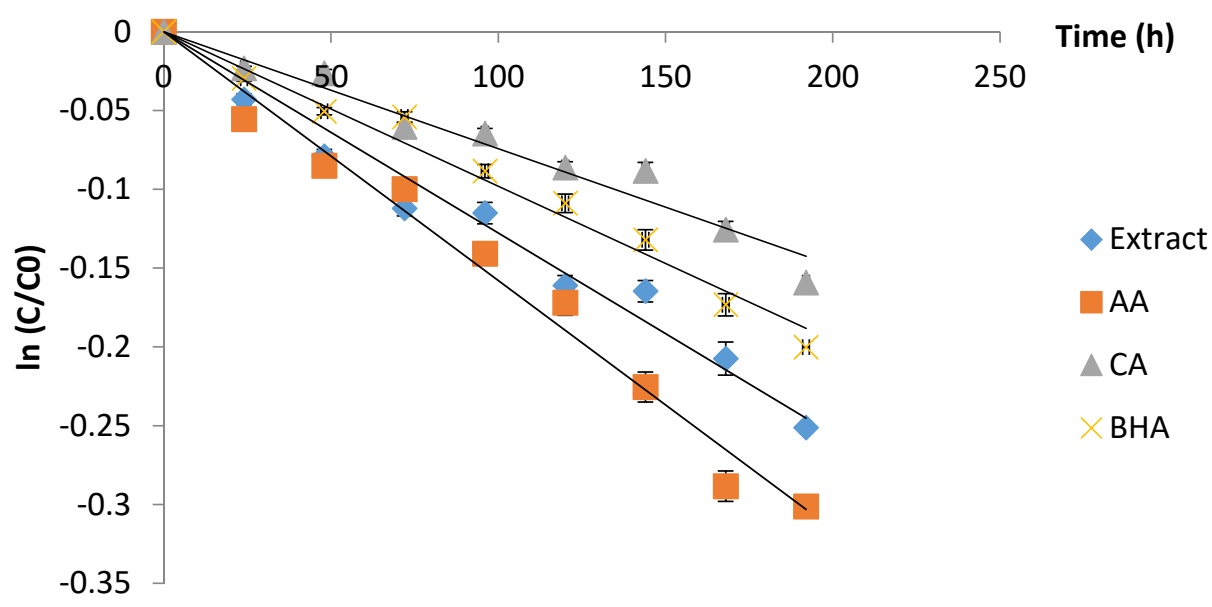

Figure 2. The degradation process of Cornelian cherries anthocyanins stored in ethanol. Error bars represent standard deviation $(n=3)$. In the some cases they are smaller than the symbol. 
The half-life values $t_{1 / 2}$ were calculated by the equation:

$$
t_{1 / 2}=\frac{-\ln 0.5}{k}
$$

The obtained values of the kinetic parameters are summarized in Table 1.

Table 1. Kinetic parameters of the degradation process of anthocyanins from Cornelian cherries

\begin{tabular}{|c|c|c|c|}
\hline Solvent/Sample & $\mathbf{k} \cdot \mathbf{1 0}^{-\mathbf{3}}\left(\mathbf{h}^{-1}\right)$ & $\mathbf{t}_{\mathbf{1 / 2}} \mathbf{( h )}$ & $\mathbf{R}^{\mathbf{2}}$ \\
\hline \multicolumn{4}{|c|}{ Water } \\
\hline Fruit extract & 0.89 & 778.65 & 0.971 \\
\hline Ascorbic acid & 4.21 & 164.61 & 0.974 \\
\hline Citric acid & 0.99 & 700 & 0.965 \\
\hline BHA & 1.55 & 447.09 & 0.972 \\
\hline \multicolumn{3}{|c|}{ Ethanol } \\
\hline Fruit extract & 1.28 & 541.41 & 0.971 \\
\hline Ascorbic acid & 1.62 & 427.78 & 0.984 \\
\hline Citric acid & 0.74 & 936.48 & 0.959 \\
\hline BHA & 0.98 & 707.14 & 0.979 \\
\hline
\end{tabular}

The kinetic rate constant was found to be higher by ethanol storage compared to water storage of anthocyanins without any added food additive. The same results were observed by investigating the degradation of grape anthocyanins which also degraded faster in alcohol than in aqueous solution [34]. Adding citric acid or BHA to the ethanolic solution of Cornelian cherry anthocyanins, resulted in a rate constant decrease to $0.74 \cdot 10^{-3} \mathrm{~h}^{-1}$ and $0.98 \cdot 10^{-3} \mathrm{~h}^{-1}$ respectively, proving the stabilizing effect of these two antioxidant additives, while AA addition resulted in a accelerate degradation process. This finding may suggest that adding $\mathrm{BHA}$ and $\mathrm{CA}$ to alcoholic beverages obtained from anthocyanin rich fruits may contribute to colour preservation of these products during storage. Regarding the Cornelian cherry anthocyanins storage in aqueous solutions, the degradation process was accelerated by the presence of $\mathrm{BHA}$ and $\mathrm{AA}$, while $\mathrm{CA}$ slightly influenced the degradation rate constant, proving that this organic acid could be the most suitable antioxidant additive to be used in juices, marmalades or compotes obtained from these fruits. 


\section{CONCLUSIONS}

The present work studied the influence of storage solvent and presence of common food antioxidant additives on the degradation process of Cornelian cherry anthocyanins at room temperature. The anthocyanins' degradation process followed a first order reaction kinetics and the rate constants ranged from $0.74 \cdot 10^{-3}$ to $4.21 \cdot 10^{-3} \mathrm{~h}^{-1}$. The results revealed that degradation in ethanol occurred $\sim 1.5$ fold faster than in water, while adding ascorbic acid dramatically destabilized the anthocyanic pigments, especially in water, where the process occurred $\sim 4.7$ fold faster in the presence of AA than in the crude extract. The addition of citric acid and BHA had a positive effect on the anthocyanins' stability in ethanol, while in water BHA accelerated the degradation process.

\section{EXPERIMENTAL SECTION}

\section{Chemicals and reagents}

All chemicals and reagents were purchased from Merck (Darmstadt, Germany), were of analytical grade and were used without further purification. A TYPDP1500 Water distiller (Techosklo LTD, Držkov, Czech Republic) was used to obtain the distilled water.

\section{Plant material}

Cornelian cherries were purchased from a local market in August 2019 in Cluj-Napoca, Romania.

\section{Extract preparation}

The extraction of the anthocyanins was carried out in acidified ethylic alcohol $96 \%(0.1 \% \mathrm{HCl})$. To this end, 50 grams of fresh fruits were grinded to obtain a fruit puree and $200 \mathrm{~mL}$ of solvent were added. The mixture was stirred for 2 hours at room temperature $\left(22^{\circ} \mathrm{C}\right)$ and then vacuum filtered. The obtained solution was concentrated by solvent evaporation under reduced pressure using a rotary evaporator (Buchi R114 Rotovap) to $83^{\circ}$ Brix. The total soluble solids expressed as ${ }^{\circ}$ Brix were determined using a digital Bellingham + Stanley RFM refractometer. In order to investigate the anthocyanin stability the obtained concentrated extract was divided in two parts.

\section{Degradation studies}

Each part of the obtained solutions was quantitatively transferred into a $250 \mathrm{~mL}$ volumetric flask and made up to $250 \mathrm{~mL}$ with distilled water and $96 \%$ ethanol, respectively. From the obtained solutions, samples were 
prepared to investigate the influence of food additives as follows: $30 \mathrm{~mL}$ anthocyanin solution (in water or ethanol) was mixed with butylated hydroxy anisole, ascorbic acid or citric acid in order to obtain a $0.1 \%(\mathrm{w} / \mathrm{v})$. final concentration of the additive in the solution, concentration usually used in the beverages and food products.

The degradation of anthocyanins in the investigated conditions was studied during 8 days by keeping samples at room temperature in the dark. All the samples were introduced in amber vials which were well capped to avoid evaporation. Immediately after preparation $(t=0)$ and after $24,48,72$, $96,120,144,168$ and 192 hours respectively, samples were collected and used to determine the total monomeric anthocyanin content. Changes in this content during storage were used to determine the degradation reaction kinetics.

\section{Determination of anthocyanin content}

Total monomeric anthocyanin content was measured according to the $\mathrm{pH}$ differential method. The samples were 8 fold diluted with acidic buffer solutions $\left(0.025 \mathrm{M} \mathrm{KCl}, \mathrm{pH}=1\right.$ and $0.4 \mathrm{M} \mathrm{CH}_{3} \mathrm{COONa}$ solution, $\left.\mathrm{pH}=4.5\right)$ and allowed to equilibrate for 15 minutes in the dark. An UV-VIS Perkin Elmer Lambda 25 double beam spectrophotometer was used to measure the absorbance of each sample at 506 and $700 \mathrm{~nm}$. From the obtained values, the total monomeric anthocyanins content was calculated and expressed as $\mathrm{mg} / \mathrm{L}$ extract in cyanidin-3-glucoside equivalents. All experiments were done in triplicate.

\section{REFERENCES}

1. E. Pojer; F. Mattivi; D. Johnson; C.S. Stockley; Compr. Rev. Food Sci. Food Saf., 2013, 12, 483-508.

2. S. Zafra-Stone; T. Yasmin; M. Bagchi; A. Chatterjee; J.A. Vinson; D. Bagchi; Mol. Nutr. Food Res., 2007, 51, 675-683.

3. A. Ben Lagha; S. Dudonne; Y. Desjardins; D. Grenier; J. Agric. Food Chem., 2015, 63, 6999-7008.

4. C. Xu; Y. Wang; H. Yu; H. Tian; X. Chen; ACS Nano, 2018, 12, 8255-8265.

5. O. Danila; A. Berghian Sevastre; V. Dionisie; D. Gheban; D. Olteanu; F. Tabaran; I. Baldea; G. Katona; B. Moldovan; S. Clichici; L. David; G. A. Filip; Nanomedicine (Lond.), 2017, 12, 1455-1473

6. A. Castaneda-Ovando; M.L. de Pacheco-Hernandez; M.E. Paez-Hernandez; J.A. Rodriguez; C.A. Galan-Vidal; Food Chem., 2009, 113, 859-871.

7. O. Rop; J.Mlcek; D.Kramarova; T.Jurikova; Afr. J. Biotechnol., 2010, 9, 1205-1210.

8. M. De Biaggi; D. Donno; M.G. Mellano; I. Riondato; E.N. Rakotoniaina; G.L. Beccaro; Plant Foods Hum. Nutr., 2018, 73, 89-94.

9. B. Moldovan; A. Popa; L. David; J. Appl. Bot. Food Qual., 2016, 89, 208-211 
10. L. David; V. Danciu; B. Moldovan; A. Filip; Antioxidants, 2019, 8, 114.

11. G. A. Filip; B. Moldovan; I. Baldea; D. Olteanu; R. Suharoschi; N. Decea; C. M. Cismaru; E. Gal; M. Cenariu; S. Clichici; L. David; J. Photochem. Photobiol. B, 2019, 191, 26-37.

12. I. Baldea; A. Florea; D. Olteanu; S. Clichici; L. David; B. Moldovan; M. Cenariu; M. Achim; R. Suharoschi; S. Danescu; G. A. Filip; Nanomedicine, 2020, 15, 55-75.

13. S. Asgary; R. Kelishadi; M. Rafieian-Kopaei; S. Najafi; M. Najafi; A. Sahebkar; Pediatr. Cardiol., 2013, 34, 1729-1735.

14. B. Dinda; A.M. Kyriakopoulos; S. Dinda; V. Zoumpourlis; N.S. Thomaidis; A. Velegraki; C. Markopoulos; M. Dinda; J. Ethnopharmacol., 2016, 193, 670-690.

15. A. Milenkovic Andelkovic; B. Radovanovic; M. Andelkovic; A. Radovanovic; V. Nikolic; V. Randelovic; Adv. Technol., 2015, 4, 26-31.

16. B. Moldovan; A. Filip; S. Clichici; R. Suharovschi; P. Bolfa; L. David; J. Funct. Foods, 2016, 26, 77-87.

17. M. Pawlowska; F. Camangi; A. Braca; Food Chem., 2010, 119, 1257-1261.

18. T. Sozanski; A.Z. Kucharska; A. Rapak; D. Szumny; M. Trocha; A. Merwid-Lad; S. Dzimira; T. Piasecki; N. Piorecki; Jan Magdalan; A. Szelag; Atherosclerosis, 2016, 254, 151-160.

19. E. Choe; D. B. Min; Compr. Rev. Food Sci. Food Saf., 2009, 8, 345-358.

20. R. Jackman; R.Y. Yada; M.A. Tung; R.A. Speers; J. Food Biochem., 1987, 11, 201 247.

21. R. Levy; Z. Okun; A. Shpigelman; Foods, 2019, 8, 207

22. M. M. Giusti; R. E. Wrolstad; Current Protocols in Food Analytical Chemistry, Wiley, New York, 2001, pp. 1-13.

23. N. Marti; A. Perez-Vicente; C. Garcia-Viguera; J. Sci. Food Agric., 2002, 82, 217-221.

24. M. Evest; L. J. Mauer; J. Agric. Food Chem., 2013, 61, 4169-4179.

25. R. Levy; Z. Okun; A. Shpiegelman; Foods, 2019, 8, 207.

26. J. Li; H. Song; N. Dong; G. Zhao; Food Sci. Biotechnol., 2014, 23, 89-96.

27. C. Garcia-Viguera; P. Bridle; Food Chem., 1999, 64, 21-26.

28. E. M. Hubbermann; A. Heins; H. Stockmann; K. Schwarz; Eur. Food Res. Technol., 2006, 223, 83-90.

29. A. Cata; I. M. C. Ienascu; C. Tanasie; M. N. Stefanut; Rev. Roum. Chim., 2019, 64, 893-899.

30. A. Wojdylo; P. Nowicka; M. Teleszko, Processes, 2019, 7, 367.

31. A. Bozdogan; K. Yasar; Turkish J. Agric. Food Sci. Technol., 2019, 7, 282-285.

32. M. M. Pragalyaashree; D. Tiroutchelvame; S. Sashikumar; J. Appl. Pharm. Sci., 2018, 8, 057-063.

33. C. C. Chen; C. Lin; M. H. Chen; P. Y. Chyang; Foods, 2019, 8, 393.

34. K. C. Tseng; H. M. Chang; J. S. B. Wu; J. Food Process Preserv., 2006, 30, 503-514. 